COMMUNICATIONS IN

ANALYSIS AND GEOMETRY

Volume 3, Number 3, 523-542, 1995

\title{
On a singular diffusion equation
}

\author{
Panagiota Daskalopoulos ${ }^{1}$ and Manuel A. Del Pino ${ }^{2}$
}

\section{Introduction.}

On $\mathbf{R}^{2}$ any metric can be expressed as $d s^{2}=e^{v}\left(d x^{2}+d y^{2}\right)$, where $x, y$ are rectangular coordinates in $\mathbf{R}^{2}$ and $v$ is a function. Let $\mathcal{R}$ denote the scalar curvature. The Ricci flow on $\mathbf{R}^{2}$ is the equation

$$
\frac{\partial}{\partial t} d s^{2}=-\mathcal{R} d s^{2}
$$

which may be rewritten as

$$
\frac{\partial v}{\partial t}=e^{-v} \Delta v
$$

or, setting $u=e^{v}$,

$$
\frac{\partial u}{\partial t}=\Delta \log u
$$

Here $\Delta$ denotes the Euclidean Laplacian. This paper is devoted to the study of the Cauchy Problem for the above equation, namely

$$
\left\{\begin{array}{cc}
\partial u / \partial t=\Delta \log u & \text { in } \mathbf{R}^{2} \times(0, T) \\
u(x, 0)=f(x) & x \in R^{2},
\end{array}\right.
$$

where $f \in L_{l o c}^{1}\left(\mathbf{R}^{2}\right)$ is non-negative and $T$ is a given positive number. By a solution we will always understand a solution in the distributional sense.

In a different setting, this equation arises as a model for long range Vander-Waals interactions in thin films of a fluid spreading on a solid surface, if certain nonlinear fourth order effects are neglected, see de Gennes [10], in [2] and the references in [8]. In that framework, $u(x, t)$ models the height of the film at the point $x$ and the instant $t$.

\footnotetext{
${ }^{1}$ Partially supported by NSF grant $445860-21170$

${ }^{2}$ Partially supported by Grants CIICT93-0323 CCE and Fondecyt 195-0303
} 
It is interesting to observe that equation (1.1) can be understood as the formal limit as $m \rightarrow 0$ of the porous-medium equation

$$
\frac{\partial u}{\partial t}=\Delta\left(\frac{u^{m}-1}{m}\right) .
$$

Notice that, formally, the case $m=0, N=2$ is critical for the porous medium equation, since $m=(N-2) / N$ defines the critical exponent in the sense of [13].

Some examples of solutions to the equation can be given explicitly, for instance

$$
u(x, t)=\frac{8(T-t)_{+}}{\left(1+|x|^{2}\right)^{2}},
$$

which represents geometrically, after stereographic projection, a family of spheres contracting eventually to a point. In addition, the globally defined solution

$$
u(x, t)=\frac{8}{\left(e^{4 t}+|x|^{2}\right)}
$$

corresponds to a family of shrinking cigar-like solitons. This solution can be obtained as a limit when $m \rightarrow 0$ of Barenblatt self-similar solutions to (1.2), see [19]. Some other interesting examples of solutions are constructed in [15].

Our goal in this paper is to characterize the solvability of problem (1.1) in terms of the initial condition $f$ and to reveal some of the very interesting and complex structure of its solution set.

Our first result provides a necessary and sufficient condition for solvability: it states in particular that there exists a solution defined up to time $T \leq+\infty$, if and only if $\int_{\mathbf{R}^{2}} f d x \geq 4 \pi T$.

Theorem 1.1. Assume that $f \not \equiv 0$. Then, there exists a solution $u$ to problem (1.1) with

$$
T=\frac{1}{4 \pi} \int_{\mathbf{R}^{2}} f(x) d x \leq+\infty .
$$

In particular, if $\int_{\mathbf{R}^{2}} f(x) d x=+\infty$, then (1.1) admits a globally defined solution. Reciprocally, if there is a solution to problem (1.1), then

$$
T \leq \frac{1}{4 \pi} \int_{\mathbf{R}^{2}} f d x .
$$

Moreover,

$$
\int_{\mathbf{R}^{2}} u(x, t) d x \leq \int_{\mathbf{R}^{2}} f d x-4 \pi t,
$$


for all $t<T$.

Thus, in particular all solutions to problem (1.1) must cease to exist by vanishing before time $(1 / 4 \pi) \int_{\mathbf{R}^{2}} f$. We note that the fact that $\int_{\mathbf{R}^{2}} f<+\infty$ implies finite extinction time was already established in [7].

On the other hand, a strong nonuniqueness phenomenon takes place as our second result shows: given any integrable initial data $f$, we may find solutions which vanish at any given time less than or equal to $(1 / 4 \pi) \int_{\mathbf{R}^{2}} f$.

Theorem 1.2. Assume $\int_{\mathbf{R}^{2}} f(x) d x<+\infty$. Then, for every $\mu>0$ there exists a solution $u_{\mu}$ to problem (1.1) for

$$
T=T_{\mu}=\frac{1}{2 \pi(2+\mu)} \int_{\mathbf{R}^{2}} f d x,
$$

with the property that

$$
\int_{\mathbf{R}^{2}} u_{\mu}(x, t) d x=\int_{\mathbf{R}^{2}} f(x) d x-2 \pi(2+\mu) t
$$

for all $t<T_{\mu}$.

It should be observed that, at least at a formal level, the number $\mu>0$ in the above constructed solutions is related to their decay rate at infinity. In fact, consider for example a solution $u(|x|, t)$ to (1.1) radially symmetric and satisfying (1.5). Then formally, if $r=|x|$, we compute

$$
\begin{aligned}
\frac{d}{d t} \int_{\mathbf{R}^{2}} u(|x|, t) d x=\int_{\mathbf{R}^{2}} & u_{t}(|x|, t) d x \\
& =\int_{\mathbf{R}^{2}} \Delta \log u(|x|, t) d x=2 \pi \lim _{r \rightarrow \infty} r(\log u)_{r}(r, t)
\end{aligned}
$$

while from (1.5) we have that

$$
\frac{d}{d t} \int_{\mathbf{R}^{2}} u(|x|, t) d x=-2 \pi(2+\mu),
$$

which roughly tells us that, at any time, $u(|x|, t)$ decays as the power $|x|^{-(2+\mu)}$.

In the same way, if for a solution of (1.1) with integrable initial data we define $\phi(t)$ by the relation

$$
\int_{\mathbf{R}^{2}} u(x, t) d x=\int_{\mathbf{R}^{2}} f d x-2 \pi \phi(t),
$$


then one has from Theorem 1.1 that $\phi(t) \geq 2 t$ and, formally, one expects $u$ to decay roughly as $|x|^{-\phi^{\prime}(t)}$ at infinity, in case that $\phi$ is differentiable. Since $u$ must remain integrable in $x$ at all times, $\phi^{\prime}(t)$ should not be less than two.

Our next result shows rigorously this fact. Moreover, we are also able to establish partially the reciprocal assertion, which generalizes Theorem 1.2 : given any function $\phi$ of class $C^{1}$ with $\phi^{\prime}(t) \geq 2$, there exists a solution $u$ so that (1.6) holds.

Theorem 1.3. Assume that $\int_{\mathbf{R}^{2}} f d x<\infty$ and that $u$ solves (1.1). Let $\phi(t)$ be the function defined by the relation (1.6). Then, for all $t \in[0, T)$ and all $h>0$ so that $t+h<T$, we have

$$
\frac{\phi(t+h)-\phi(t)}{h} \geq 2 .
$$

Reciprocally, if $\phi:[0, T] \rightarrow[0, \infty)$ is any continuously differentiable function such that $\phi^{\prime}(t) \geq 2, \phi(0)=0$ and $\phi(T)=(1 / 2 \pi) \int_{\mathbf{R}^{2}} f$, then there exists a solution $u$ to (1.1) such that (1.6) holds.

Even though smoothness of $\phi$ can be relaxed in the construction of these solutions, see the Remark at the end of $\S 4$, some regularity of the given $\phi$ seems to be needed.

Finally, we mention that existence and long time behavior for this equation under different assumptions on the initial data, including slower, nonintegrable decay, have been studied in [18], [19]. The one dimensional case has been treated in [9], [16] and [15]. A priori bounds and decay estimates in the higher dimensional case have been derived in [8].

In the rest of the paper we will prove the above results. In $\S 3$ we prove the basic results, Theorems 1.1 and 1.2. Our most general result, Theorem 1.3 is established in $\S 4$. For existence the comparison with certain special traveling wave-like solutions found in [11] is crucial, and we review them in $\S 2$.

Acknowledgement. We are grateful to Richard Hamilton for many useful suggestions and enlightening discussions in the course of this work.

\section{Construction of some special solutions.}

In this section, we review the construction of certain special, traveling wave-like solutions to equation (1.1), which will be of main assistance in the proof of the theorems. Since this analysis is essentially contained in [11], we will be sketchy. The main result of this section is the following: 
Proposition 2.1. Given any numbers $K>0$ and $\mu>0$, there exists a radially symmetric, smooth, positive solution $v(x, t)$ to equation (1.1) with $T=K / 2 \pi(2+\mu)$ such that

$$
\int_{\mathbf{R}^{2}} v(x, t) d x=K-2 \pi(2+\mu) t \quad \forall t \in[0, T) .
$$

Proof. For the proof it suffices to show that for each $\mu>0$, there is a radially symmetric solution $w(|x|, t)$ to $(1.1)$ with $T=1$, which satisfies

$$
\int_{0}^{\infty} w(r, t) r d r=(1-t)(2+\mu)
$$

Indeed, if $w$ is such a solution, then

$$
v(x, t)=(K / 2 \pi(2+\mu)) w(|x|,(2 \pi(2+\mu) / K) t)
$$

is a solution to (1.1) which satisfies (2.1). When $\mu=2$, we just take the explicit solution

$$
w(r, t)=\frac{8(1-t)}{\left(1+r^{2}\right)^{2}}
$$

Assume next that $\mu>2$. We look for a solution $w$ of the following form:

$$
w(|x|, t)=(1-t) \frac{g(\log |x|-\gamma \log (1-t))}{|x|^{2}}
$$

where $\gamma>0$ is to be determined in terms of $\mu$. A direct computation shows that then $g$ must satisfy the differential equation

$$
\left\{\frac{g^{\prime}}{g}\right\}^{\prime}+g-\gamma g^{\prime}=0, \quad y \in(-\infty, \infty)
$$

This equation has been analyzed in [11]. In particular, it is shown there that given any numbers $\alpha>\beta>0$, there is a choice of $\gamma>0$ such that there exists a solution $g(y)$ to (2.4) which admits expansions of the form $g(y)=a_{1} e^{-\alpha y}+a_{2} e^{-2 \alpha y}+\cdots$ as $y \rightarrow+\infty$ and $g(y)=b_{1} e^{\beta y}+b_{2} e^{2 \beta y}+\cdots$ as $y \rightarrow-\infty$.

Let us choose $\beta=2$ and $\alpha=\mu$. Note that this choice makes the associated $w$ in (2.3) nonsingular and a solution to (1.1) up to $x=0$. Moreover, we have that $\left(g^{\prime} / g\right)(+\infty)=-\mu$ and $\left(g^{\prime} / g\right)(-\infty)=+2$. Hence, integrating (2.4) we obtain that

$$
2+\mu=\int_{-\infty}^{\infty} g(y) d y
$$


from where relation (2.2) readily follows.

Finally, assume $0<\mu<2$. In this case we take $\alpha=2$ and $\beta=-\mu$ and choose $\gamma>0$ and $g$ as above. Let us now set

$$
w(|x|, t)=(1-t) \frac{g(-\log |x|-\gamma \log (1-t))}{|x|^{2}} .
$$

This $w$ defines a solution of (1.1) with the desired properties, thus finishing the proof.

\section{Proofs of Theorems 1.1 and 1.2 .}

In this section we present the proofs of Theorems 1.1 and 1.2. We will first give the proof of Theorem 1.2, as the proof of the existence assertion in Theorem 1.1 is going to be based on Theorem 1.2.

Proof of Theorem 1.2. As we mentioned in the introduction, solutions which satisfy (1.5) are expected, at least formally, to decay at infinity as the power $|x|^{-(2+\mu)}$. Having this in mind, our construction goes as follows : for a given $\mu$, we solve the boundary value problem

$$
\left\{\begin{array}{cc}
\partial u / \partial t=\Delta \log u & \text { in } B_{R} \times[0, \infty) \\
u(x, t)=R^{-(2+\mu)} & \text { on } \partial B_{R} \times(0, \infty) \\
u(x, 0)=f(x) & x \in B_{R} .
\end{array}\right.
$$

on a sequence of expanding cylinders $B_{R_{n}} \times[0, \infty)$ and then use the spesific solutions of $\S 2$ as barriers in an average sense to show that the limit of those solutions along a subsequence of $\left\{R_{n}\right\}$ is a solution which satisfies (1.5).

Before we begin with our construction, we introduce the operator

$$
G_{R}^{*}(h)(x)=\int_{\mathbf{R}^{2}}\left[G_{R}(x, y)-G_{R}(0, y)\right] h(y) d y,
$$

where for $R>0, G_{R}(x, y)$ denotes the Greens function for the ball $B_{R}=$ $\{x ;|x| \leq R\}$, given by

$$
\left\{\begin{array}{c}
\frac{1}{2 \pi}\left\{\log (|x-y|)-\log \left(\left|\frac{|x| y}{R}-\frac{R x}{|x|}\right|\right)\right\}, \quad \text { if } x \neq 0 \\
\frac{1}{2 \pi}\{\log (|y|)-\log (R)\}, \quad \text { if } x=0
\end{array}\right.
$$


Notice that if $h$ is bounded,

$$
\Delta G_{R}^{*}(h)(x)=h(x), \quad \forall x \in B_{R}
$$

while if $h$ is smooth and constant on $\partial B_{R}$, one has

$$
G^{*}(\Delta h)(x)=h(x)-h(0), \quad \forall x \in B_{R} .
$$

We will first show the result under the assumption that the initial data $f$ is in $C_{0}^{\infty}\left(\mathbf{R}^{2}\right)$. The general case will follow by approximation.

To simplify the notation we will omit the subscript $\mu$ from $u$ and $T$. For $R>0$ and $\epsilon \in(0,1)$, let $u_{\epsilon}^{R}$ denote the unique solution to the boundary value problem (3.1) with initial data $u_{\epsilon}^{R}(x, 0)=f(x)+\epsilon$, instead of $f(x)$. Existence and uniqueness of $u_{\epsilon}^{R}$ follow from the standard theory of nondegenerate quasilinear parabolic equations; see for example in [14].

Consider $G_{R}^{*}(f)$, as defined in (3.2). We claim that if the support of $f$ is contained in the ball $B_{R_{0}}$, then for all $R \geq R_{0}$ and all $x \in B_{R}$, we have

$$
G_{R}^{*}(f)(x)=\log (|x|+1) \int_{\mathbf{R}^{2}} f d x+\theta(x),
$$

where the function $\theta(x)$ is locally bounded with

$$
\theta(x)=o(\log (|x|+1)), \quad \text { as }|x| \rightarrow \infty .
$$

Indeed, we write

$$
G_{R}^{*}(f)(x)=\frac{1}{2 \pi}\left\{\int_{B_{R_{0}}} \log \left(\frac{|x-y|}{|y|}\right) f(y) d y+\int_{B_{R_{0}}} k_{R}(x, y) f(y) d y\right\}
$$

where

$$
\left|k_{R}(x, y)\right|=\left|\log \left(\left|\frac{|x| y}{R^{2}}-\frac{x}{|x|}\right|\right)\right| \leq 2 \frac{|y||x|}{R^{2}} \leq 1,
$$

for $y \in B_{R_{0}}$ and $x \in B_{R}$, if $R \geq 2 R_{0}$. Therefore

$\theta(x)=\frac{1}{2 \pi}\left\{\int_{B_{R_{0}}}\left[\log \left(\frac{|x-y|}{|y|}\right)-\log (|x|+1)\right] f(y) d y+\int_{B_{R_{0}}} k_{R}(x, y) f(y) d y\right\}$, satisfies $\lim _{|x| \rightarrow \infty} \theta(x) / \log (|x|+1)=0$.

For $\delta>0$ small, let $v^{T-\delta}, v^{T+\delta}$ denote the specific radially symmetric solutions constructed in $\S 2$ and satisfying

$$
\int_{\mathbf{R}^{2}} v^{T-\delta}(x, 0) d x=2 \pi(2+\mu)(T-\delta), \quad \int_{\mathbf{R}^{2}} v^{T+\delta}(x, 0) d x=2 \pi(2+\mu)(T+\delta) .
$$


Since $\int_{\mathbf{R}^{2}} f d x=2 \pi(2+\mu) T$, it follows from (3.5) and (3.6) that

$$
-l_{\delta}+G_{R}^{*}\left(v^{T-\delta}(\cdot, 0)\right)(x) \leq G_{R}^{*}(f)(x) \leq G_{R}^{*}\left(v^{T+\delta}(\cdot, 0)\right)(x)+l_{\delta},
$$

for some nonnegative constant $l_{\delta}$.

We would like to show that if $u^{R}=\lim _{\epsilon \rightarrow 0} u_{\epsilon}^{R}$, then there exists a sequence $R_{j} \uparrow \infty$ and a nonnegative constant $L_{\delta}$, which can be chosen to be independent of $R_{j}$, such that

$$
\begin{aligned}
-L_{\delta}+G_{R_{j}}^{*}\left(v^{T-\delta}(\cdot, t)\right) & (x) \\
& \leq G_{R_{j}}^{*}\left(u^{R_{j}}(\cdot, t)(x) \leq G_{R_{j}}^{*}\left(v^{T+\delta}(\cdot, 0)\right)(x)+L_{\delta} .\right.
\end{aligned}
$$

for all $|x| \leq R_{j}$ and $0 \leq t \leq T-2 \delta$. We begin by showing the left hand side of (3.7). Set

$$
W(x, t)=G_{R}^{*}\left(u_{\epsilon}^{R}(\cdot, t)-v^{T-\delta}(\cdot, t)\right)(x) .
$$

Using the maximum principle we will prove that $W(x, t) \geq-L_{\delta}$, for $|x| \leq R$, $0 \leq t \leq T-2 \delta$. Indeed, since both functions $u_{\epsilon}^{R}$ and $v^{T-\delta}$ are constant on $\partial B_{R}$, we can compute using (3.4) that

$$
\frac{\partial W}{\partial t}=G_{R}^{*}\left(\Delta\left[\log u_{\epsilon}^{R}-\log v^{T-\delta}\right]\right)=a(x, t) \Delta W-b(t),
$$

where

$$
a(x, t)=\frac{\log u_{\epsilon}^{R}-\log v^{T-\delta}}{u_{\epsilon}^{R}-v^{T-\delta}}
$$

and

$$
b(t)=\log u_{\epsilon}^{R}(0, t)-\log v^{T-\delta}(0, t) \leq B,
$$

with $B$ depending only on $\|f\|_{\infty}$ and $\delta$. Therefore, if we set $\widetilde{W}=W+B t$, then $\widetilde{W}$ satisfies the differential inequality

$$
\frac{\partial \widetilde{W}}{\partial t} \geq a(x, t) \Delta \widetilde{W}
$$

on $B_{R} \times(0, T-\delta)$. At $t=0$ we have

$$
\widetilde{W}(x, 0)=W(x, 0) \geq-l_{\delta}
$$

To see what happens on the lateral boundary, we first observe that for the special solution $v^{T-\delta}$ there exist constants $c_{1}(\delta)$ and $c_{2}(\delta)$, such that for $0 \leq t \leq T-2 \delta$,

$$
\frac{c_{1}(\delta)}{R^{2+\mu}} \leq v^{T-\delta}(R, t) \leq \frac{c_{2}(\delta)}{R^{2+\mu}}
$$


if $R \geq R_{0}$, with $R_{0}$ sufficiently large. It follows that for $|x|=R \geq R_{0}$, $0 \leq t \leq T-2 \delta$

$$
\frac{\partial \widetilde{W}(x, t)}{\partial t}=\log u_{\epsilon}^{R}(x, t)-\log v^{T-\delta}(x, t)-b(t)+B \geq 0,
$$

if $B$ is chosen sufficiently large, which implies that for $|x|=R, 0 \leq t \leq T-2 \delta$ we have

$$
\widetilde{W}(x, t) \geq W(x, 0) \geq-l_{\delta} .
$$

We can apply now the maximum principle to conclude that $\widetilde{W}(x, t) \geq-l_{\delta}$. Hence, by letting $\epsilon \rightarrow 0$ we obtain for $0 \leq t \leq T-2 \delta$ the inequality

$$
\left.G_{R}^{*}\left(u^{R}(\cdot, t)\right)(x) \geq G_{R}^{*}\left(v^{T-\delta}\right)(\cdot, t)\right)(x)-L_{\delta},
$$

with $L_{\delta}=l_{\delta}+B T$. Before we show the right hand side of (3.7) we will first construct the solution $u$. Taking spherical averages on both sides of (3.9) we obtain

$$
\int_{0}^{r} \frac{d s}{2 \pi s} \int_{B_{s}} u^{R}(x, t) d x \geq \int_{0}^{r} \frac{d s}{2 \pi s} \int_{B_{s}} v^{T-\delta}(x, t) d x-L_{\delta}
$$

for $0<r<R$ and $0<t \leq T-2 \delta$. Remembering that the special solutions $v^{T-\delta}$ have the form

$$
v^{T-\delta}(x, t)=(T-t-\delta)_{+} \frac{g(\log |x|+\log \alpha(t))}{|x|^{2}},
$$

with $\alpha(t)=((T-\delta) /(T-t-\delta))^{\gamma}$ and $\int g(y) d y=2+\mu$, and computing

$$
\int_{0}^{r} \frac{d s}{2 \pi s} \int_{B_{s}} v^{T-\delta}(x, t) d x=(T-t-\delta)_{+} \int_{0}^{r \alpha(t)} \frac{d s}{2 \pi s} \int_{B_{s}} g(\log |x|) d x,
$$

we deduce that by choosing $L_{\delta}$ sufficiently large we can make

$$
\int_{0}^{r} \frac{d s}{2 \pi s} \int_{B_{s}} v^{T-\delta}(x, t) d x \geq(2+\mu)(T-t-2 \delta) \log (1+r)-L_{\delta},
$$

for all $r>0$. Therefore, we have

$$
\int_{0}^{r} \frac{d s}{2 \pi s} \int_{B_{s}} u^{R}(x, t) d x \geq(2+\mu)(T-t-2 \delta) \log (1+r)-L_{\delta} .
$$

Basic Claim : Given a number $\delta>0$ and an increasing sequence $R_{j} \uparrow \infty$, there exists a subsequence, still denoted by $\left\{R_{j}\right\}$, such that the sequence of 
solutions $u^{R_{j}}$ converges uniformly on compact subsets of $\mathbf{R}^{2} \times(0, T-3 \delta]$ to a solution $u^{\delta}$ of (1.1).

To show the claim, we begin by observing that there exists a point $x_{0} \in$ $\mathbf{R}^{2}$ such that

$$
\limsup _{R_{j} \rightarrow \infty} u^{R_{j}}\left(x_{0}, T-2 \delta\right)>0 .
$$

Indeed, if $\lim _{j \rightarrow \infty} u^{R_{j}}(x, T-2 \delta)=0$, for all $x \in \mathbf{R}^{2}$, then from Dominated convergence we conclude that

$$
\limsup _{n \rightarrow \infty} \int_{0}^{r} \frac{d s}{2 \pi s} \int_{B_{s}} u^{R_{j}}(x, T-2 \delta) d x=0
$$

for all $r>0$, which contradicts (3.10). It follows from (3.11) that we can choose a subsequence, still denoted by $\left\{R_{j}\right\}$, such that

$$
u^{R_{j}}\left(x_{0}, T-2 \delta\right) \geq c>0 \quad \forall j \geq j_{0} .
$$

For $r>0$ and $s \in(0, T-3 \delta)$ set $K=B_{r}\left(x_{0}\right) \times(s, T-3 \delta]$ and let $j \geq j_{0}$ be sufficiently large so that the cylinder $B_{R_{j}} \times(0, T)$ strictly contains $K$. We will show that (3.12) implies the estimate from below

$$
u^{R_{j}}(y, \tau) \geq c(K)>0 \quad \forall j \geq j_{0}, \quad(y, \tau) \in K .
$$

This will be a consequence of the following Harnack type estimates, satisfied by all solutions $u^{R_{j}}$,

$$
(\pi \rho)^{-2} \int_{B_{\rho}\left(y_{0}\right)}(-\log u)(x, t) d x \leq(-\log u)\left(y_{0}, T-2 \delta\right)-\log t+C_{\delta} M \rho^{2}
$$

and

$$
(-\log u)\left(y_{0}, t\right) \leq(\pi \rho)^{-2} \int_{B_{\rho}\left(y_{0}\right)}(-\log u)(x, t) d x-\frac{M \rho^{2}}{t},
$$

holding for all $\rho>0$ such that $B_{\rho}\left(y_{0}\right) \subset \subset B_{R_{j}}(0)$ and $t \in(0, T-3 \delta]$. Here $M$ is an upper bound for the initial data $f$. The estimate (3.14) is proven in [17] (Lemma 6) and the estimate (3.15) can be easily proven in a similar manner, by multiplying the inequality $\Delta \log u \leq 1 / t u$ by the Green's function used in [17] and integrating. From (3.12) and (3.14) we obtain

$$
\int_{B_{\rho}\left(x_{0}\right)}\left(-\log u^{R_{j}}\right)(x, t) d x \leq C(\rho, \delta)-\log t, \quad 0<t \leq T-3 \delta .
$$


Thus if $(y, \tau) \in K$ and $\rho=2 r,(3.15)$ and (3.16) imply that

$$
\left(-\log u^{R_{j}}\right)(y, \tau) \leq C(K) \int_{B_{\rho}\left(x_{0}\right)}\left(-\log u^{R_{j}}\right)(x, \tau) d x \leq C(K),
$$

which yields (3.13).

Since the sequence of solutions $\left\{u^{R_{j}}\right\}$ is uniformly bounded from above by $\|f\|_{\infty}$, it follows from (3.12) and the classical theory of parabolic equations that the sequence $\left\{u^{R_{j}}\right\}$ is equicontinuous on compact subsets of $\mathbf{R}^{2} \times(0, T-3 \delta]$. Hence, there exists a subsequence, still denoted by $\left\{u^{R_{j}}\right\}$, which converges uniformly on compact subsets of $\mathbf{R}^{2} \times(0, T-3 \delta]$ to a function $u^{\delta}$. It remains to show that $u^{\delta}$ is a solution of $(1.1)$ on $\mathbf{R}^{2} \times(0, T-3 \delta]$. It is clear that $u^{\delta}$ satisfies the equation (1.1) in the distributional sense, since each $u^{R_{j}}$ does. Also, because $u^{\delta} \leq\|f\|_{\infty}$, it follows from (3.16) that $\log u^{\delta} \in L_{l o c}^{1}\left(\mathbf{R}^{2} \times[0, T-3 \delta]\right)$, with

$$
\int_{B_{r}(0)}\left|\log u^{\delta}(x, t)\right| d x \leq C\left(\|f\|_{\infty}, r, \delta\right)|\log t| .
$$

It remains to show that $u^{\delta}(\cdot, \tau) \rightarrow f$ in $L_{\text {loc }}^{1}\left(\mathbf{R}^{2}\right)$, as $\tau \rightarrow 0$. Indeed, observe first that for $r>0$, (3.16) implies that

$$
\left|\int_{B_{r}(0)} u^{\delta}(x, \tau) d x-\int_{B_{r}(0)} f(x) d x\right| \leq C(\delta, r) \int_{0}^{\tau}|\log t| d t .
$$

Moreover, if for $\epsilon>0, u_{\epsilon}$ denotes the unique solution to (1.1) with initial data $f_{\epsilon}=f+\epsilon$, then $u^{\delta} \leq u_{\epsilon}$ (see in [7], Theorem 1.2) and therefore we have

$$
\begin{aligned}
\int_{B_{r}(0)}\left[u^{\delta}(x, \tau)-f(x)\right]_{+} d x \leq & \int_{B_{r}(0)}\left[u_{\epsilon}(x, t)-f(x)\right]_{+} \\
& \leq \int_{B_{r}(0)}\left|u_{\epsilon}(x, t)-f_{\epsilon}(x)\right| d x+\left|B_{r}(0)\right| \epsilon .
\end{aligned}
$$

We then easily conclude that

$$
\int_{B_{r}(0)}\left|u^{\delta}(x, \tau)-f(x)\right| d x \rightarrow 0, \quad \text { as } \tau \rightarrow 0
$$

which proves the desired result.

In order to construct a solution which is defined up to time $T$ and satisfies (3.1) we will combine (3.10) with the following estimate from above

$$
\int_{0}^{r} \frac{d s}{2 \pi s} \int_{B_{s}} u^{\delta}(x, t) d x \leq(2+\mu)(T-t+2 \delta) \log (r+1)+L_{\delta},
$$


holding for all $0<r<R$ and $0<t \leq T-2 \delta$. It is easy to observe that (3.18) follows from the right hand side of (3.7) by taking spherical averages, computing

$$
\int_{0}^{r} \frac{d s}{2 \pi s} \int_{B_{s}} v^{T+\delta}(x, t) d x \leq(2+\mu)(T-t+2 \delta) \log (r+1)+L_{\delta},
$$

for all $r>0$ and letting $R_{j} \rightarrow \infty$. Hence, we need to show the right hand side of (3.7). We will use again the maximum principle. As before, we will apply the maximum principle to the function

$$
Z(x, t)=G_{R}^{*}\left(u_{\epsilon}^{R}(\cdot, t)-v^{T+\delta}\right)(x),
$$

which satisfies the equation $\partial Z / \partial t=d(x, t) \Delta Z-e(t)$ with

$$
e(t)=\log u_{\epsilon}^{R_{j}}(0, t)-\log v^{T+\delta}(0, t) .
$$

To bound the coefficient $e(t)$, we notice that from (3.13), $u_{\epsilon}^{R_{j}}(0, T-2 \delta) \geq c$, for some positive constant $c$ depending only on $\delta$. Moreover, each of the solutions $u_{\epsilon}^{R_{j}}$ satisfies the Aronson - Bénilan inequality $u_{t} \leq 1 / t u$, which by integration yields

$$
\log u_{\epsilon}^{R_{j}}(0, t) \geq \log u_{\epsilon}^{R_{j}}(0, T-2 \delta)-\log (T-2 \delta)+\log t \geq C(\delta)+\log t .
$$

Hence, $e(t) \geq-E+\log t$, for some constant $E=E(\delta)$ and thus is we set $E(t)=E t+\int_{0}^{t} \log s d s$ and $\widetilde{Z}=Z+E(t)$, then $\widetilde{Z}$ satisfies

$$
\frac{\widetilde{\partial} Z}{\widetilde{\partial} t} \leq d(x, t) \Delta Z
$$

At $t=0$ we have $\widetilde{Z}(x, 0)=Z(x, 0) \leq l_{\delta}$. Moreover, by choosing the constant $E$ sufficiently large we can show as we did for $\widetilde{W}$ that $\partial \widetilde{Z} / \partial t \leq 0$ on $|x|=R$, and conclude that $Z \leq l_{\delta}$ on the lateral boundary of $B_{R}(0) \times(0, T-2 \delta)$. Therefore, by the maximum principle $\widetilde{Z} \leq l_{\delta}$, which implies the right hand side of (3.7) for $R=R_{j}$, and $L_{\delta}$ sufficiently large.

We have shown that the solution $u^{\delta}$ satisfies

$$
\begin{aligned}
-L_{\delta}+(2+\mu)(T-t-2 \delta) & \leq \frac{1}{\log (r+1)} \int_{0}^{r} \frac{d s}{2 \pi s} \int_{B_{s}} u^{\delta}(x, t) d x \\
& \leq(2+\mu)(T-t+\delta) L_{\delta}
\end{aligned}
$$


for all $r>0$ and $0 \leq t \leq T-2 \delta$. A simple computation shows that if $h$ is a bounded integrable function then

$$
\lim _{r \rightarrow \infty} \frac{1}{\log (r+1)} \int_{0}^{r} \frac{d s}{2 \pi s} \int_{B_{s}} h(x) d x \rightarrow \frac{1}{2 \pi} \int_{\mathbf{R}^{2}} h(x) d x .
$$

Thus setting $u_{k}=u^{\delta} \chi_{|x| \leq k}$ and using the right hand side of (3.19) we can deduce that

$$
\int_{|x| \leq k} u_{k}(x, t) d x \leq 2 \pi(2+\mu)(T-t-2 \delta),
$$

for $0 \leq t \leq T-2 \delta$. It follows by monotone convergence that $\int_{\mathbf{R}^{2}} u^{\delta}(x, t) d x<$ $\infty$ and therefore we can use again (3.19) to conclude that

$$
(2+\mu)(T-t-\delta) \leq \frac{1}{2 \pi} \int_{\mathbf{R}^{2}} u^{\delta}(x, t) d x \leq(2+\mu)(T-t+2 \delta) .
$$

It remains to construct a solution $u$ of the problem (1.1) which is defined up to time $T$ and satisfies (1.5). Choose a decreasing sequence $\delta_{k} \downarrow 0$ and starting with any increasing sequence $R_{j} \uparrow \infty$, let $R_{j}^{1}$ be a subsequence such that $u^{R_{j}^{1}} \rightarrow u^{\delta_{1}}$ uniformly on compacts of $\mathbf{R}^{2} \times\left(0, T-3 \delta_{1}\right]$. We construct $u^{\delta_{k}}$ inductively. Suppose $u^{\delta_{k-1}}=\lim _{R_{j}^{k-1} \rightarrow \infty} u^{R_{j}^{k-1}}$, then let $R_{j}^{k}$ be a subsequence of $R_{j}^{k-1}$ such that $u^{R_{j}^{k}}$ converges uniformly on compact subsets of $\mathbf{R}^{2} \times\left(0, T-3 \delta_{k}\right]$ and set $u^{\delta_{k}}=\lim _{R_{j}^{k} \rightarrow \infty} u^{R_{j}^{k}}$. By construction we have $u^{\delta_{k}}=u^{\delta_{k-1}}$ on $\mathbf{R}^{2} \times\left(0, T-3 \delta_{k-1}\right)$ and therefore if we define $u(x, t)=u^{\delta_{k}}(x, t)$, if $0<t \leq T-3 \delta_{k}$, it is clear that $u$ is the desired solution defined on $\mathbf{R}^{2} \times(0, T)$. Moreover, it follows from (3.20) that $u$ satisfies the important idendity (1.5).

To remove the assumption that $f \in C_{0}^{\infty}$, choose an increasing sequence $f_{k} \in C_{0}^{\infty}$, such that $\left\|f_{k}-f\right\|_{L^{1}\left(\mathbf{R}^{2}\right)} \rightarrow 0$ and let $u_{k}$ be the solution of the problem (1.1) defined on $\mathbf{R}^{2} \times\left(0, T_{k}\right), T_{k}=1 /(2 \pi(2+\mu)) \int_{\mathbf{R}^{2}} f_{k}$, as constructed above. Eack of the $u_{k}$ satisfies

$$
\int_{\mathbf{R}^{2}} u_{k}(x, t) d x=\int_{\mathbf{R}^{2}} f_{k}(x) d x-2 \pi(2+\mu) t, \quad 0 \leq t \leq T_{k} .
$$

Moreover, from the previous construction it is easy to deduce that the sequence $u_{k}$ is increasing. Therefore if we set

$$
u(x, t)=\lim _{k \rightarrow \infty} u_{k}(x, t), \quad \text { for }(x, t) \in \mathbf{R}^{2} \times(0, T),
$$


we can easily conclude from (3.21) and monotone convergence that $u(\cdot, t) \in$ $L^{1}\left(\mathbf{R}^{2}\right)$, for all $t \in(0, T)$ and satisfies (3.1). Moreover, since $(\log u)_{+} \leq u$ and $u \geq u_{k}$, we have that $\log u \in L_{l o c}^{1}\left(\mathbf{R}^{2} \times[0, T)\right)$. It follows in a similar manner as above, that $u$ satisfies (1.1) in the sense of distributions and that $u(\cdot, \tau) \rightarrow f$ in $L_{l o c}^{1}\left(\mathbf{R}^{2}\right)$, as $\tau \rightarrow 0$. The proof of the theorem is now complete.

We continue with the proof of Theorem 1.1.

Proof of Theorem 1.1. The proof of the nonexistence part of the Theorem is essentially contained in [7]. For the sake of completeness, we provide a proof just in the case that $f$ is uniformly bounded. The general case follows by approximation, as in [7].

For $\varepsilon>0$, we let $u_{\varepsilon}$ denote the solution of problem (1.1) with initial data $f+\varepsilon$. Standard arguments show that this solution is unique and globally defined in time. Moreover, $u_{\varepsilon} \geq u$ for all $\varepsilon>0$ (see in [7], Theorem 1.2). We will estimate $u_{\varepsilon}$. Denote

$$
N(g)(r)=\frac{1}{2 \pi} \int_{0}^{r} \frac{1}{s} \int_{|x| \leq s} g(x) d x
$$

and observe that if $\bar{g}(r)$ denotes the spherical averages of $g$, then

$$
N(\Delta g)(r)=\bar{g}(r)-g(0) .
$$

Thus, applying the operator $N$ to the equation and then integrating in time on $[0, T-\delta]$, we obtain

$$
\begin{aligned}
-\int_{0}^{T-\delta} \overline{\log u_{\varepsilon}}(r, t) d t+\int_{0}^{T-\delta} & \log u_{\varepsilon}(0, t) d t \\
& =-N\left(u_{\varepsilon}(\cdot, T-\delta)\right)(r)+N(f+\varepsilon)(r) .
\end{aligned}
$$

Now, since $u_{\varepsilon}$ satisfies the Aronson-Bénilan inequality, $u_{t} / u \leq 1 / t$, we have that

$$
-\int_{0}^{T-\delta} \log u_{\varepsilon}(0, t) d t \leq-(T-\delta) \log u_{\varepsilon}(0, T-\delta)-\int_{0}^{T-\delta} \log \left(\frac{s}{T-\delta}\right) d s
$$

Using (3.22), (3.23) and Jensen's inequality we get

$$
\begin{aligned}
-\int_{0}^{T-\delta} \log \bar{u}_{\varepsilon}(r, s) d s \leq N(f) & (r)+C^{*}+A \varepsilon r^{2} \\
\leq & \frac{\log r}{2 \pi} \int_{\mathbf{R}^{2}} f d x+o(\log r)+A \varepsilon r^{2}
\end{aligned}
$$


where the constant $C^{*}$ is given by the right hand side of (3.23). Since $u_{\varepsilon}(0, T-\delta)>u(0, T-\delta)>0$, we can choose the constant $C^{*}$ to be independent of $\varepsilon$. Thus, from (3.24) and the Aronson-Bénilan inequality we deduce that

$$
\bar{u}_{\epsilon}(r, \delta) \geq B r^{1 /(2 \pi(T-2 \delta))} \int_{\mathbf{R}^{2}} f \exp \left(-A \varepsilon r^{2}-C\right),
$$

with constants $B$ and $C$ independent of $\delta$ and $\varepsilon$. On the other hand applying again the operator $N$ to the equation and integrate in time on $[0, \delta]$, we obtain

$$
\int_{0}^{\delta} \overline{\log u_{\varepsilon}}(r, t) d t \geq \int_{0}^{\delta} \log u_{\varepsilon}(0, t) d t+N\left(\bar{u}_{\varepsilon}(\cdot, \delta)\right)(r)-N(f+\epsilon)(r),
$$

and therefore with the use of (3.25) we deduce the inequality

$$
\int_{0}^{\delta} \overline{\log u_{\varepsilon}}(r, t) d t \geq B N\left(r^{1 /(2 \pi(T-2 \delta))} \int f d x \exp \left(-A \varepsilon r^{2}-C\right)\right)-C^{*}-A \epsilon r^{2},
$$

where $C$ and $C^{*}$ can be taken independent of $\varepsilon$. Since the initial data $f$ is uniformly bounded, the left hand side of the above inequality is uniformly bounded independently of $\epsilon$. Therefore we must have

$$
\frac{1}{2 \pi(T-2 \delta)} \int_{\mathbf{R}^{2}} f d x \geq 2 .
$$

Since $\delta$ is arbitrary the result follows in case that $f$ is bounded. In the general case, one proceeds using approximations for $f$ which are bounded, as in [7].

We will show next (1.4). Assume that there is a $\bar{t} \in(0, T)$ such that the opposite inequality holds, i.e.,

$$
\int_{\mathbf{R}^{2}} u(x, \bar{t}) d x>\int_{\mathbf{R}^{2}} f d x-4 \pi \bar{t} .
$$

From Theorem 1.2 we know that for every $\mu>0$ there exists a solution $v$ to (1.1) defined on $\left[\bar{t}, \bar{t}+T_{\mu}\right]$, whose initial data is $u(\cdot, \bar{t})$, and where

$$
T_{\mu}=\frac{1}{2 \pi(2+\mu)} \int_{\mathbf{R}^{2}} u(x, \bar{t}) d x .
$$

Thus, the function $w$ defined on $\left[0, \bar{t}+T_{\mu}\right]$ by matching together $u$ and $v$ is a solution to (1.1) in this interval, with initial data $f$. But because of (3.25), by choosing $\mu$ sufficiently small we can make

$$
\bar{t}+T_{\mu}>\frac{1}{4 \pi} \int_{\mathbf{R}^{2}} f(x) d x
$$


which contradicts (1.3). Therefore (1.4) must hold true.

We will now construct a solution with the given initial data, which exists up to time $T=1 / 4 \pi \int_{\mathbf{R}^{2}} f(x) d x$. This is going to be the maximal solution with the given initial data, as actually (1.3) indicates. Its construction is fairly straightforward. As in the proof of Theorem 1.2, we make first the extra assumption that $f \in C_{0}^{\infty}\left(\mathbf{R}^{2}\right)$. For $\epsilon>0$, let $u^{\epsilon}$ denote the solution of the initial value problem (1.1) with initial data $u^{\epsilon}(x, 0)=f+\epsilon$, defined on $\mathbf{R}^{2} \times(0, \infty)$. Then, if for $\mu>0, u_{\mu}$ denotes the solution constructed above and satisfying (1.6), we have

$$
u_{\mu} \leq u^{\epsilon} \quad \text { on } \mathbf{R}^{2} \times\left(0, T_{\mu}\right) .
$$

As it follows by the maximum principle that the sequence $\left\{u^{\epsilon}\right\}$ is monotonic and hence the limit $u=\lim _{\epsilon \rightarrow 0} u^{\epsilon}$ exists and for all $\mu>0$ satisfies

$$
u_{\mu} \leq u \quad \text { on } \mathbf{R}^{2} \times\left(0, T_{\mu}\right)
$$

Since $T_{\mu} \uparrow T$, as $\mu \rightarrow 0$, it is easy to conclude from (3.27) that $u$ is a solution of (1.1) on $\mathbf{R}^{2} \times(0, T)$, with $T=1 / 4 \pi \int_{\mathbf{R}^{2}} f(x) d x$. Moreover (1.5) satisfied by all $u_{\mu}, \mu>0$ implies that

$$
\int_{\mathbf{R}^{2}} u(x, t) d x \geq \int_{\mathbf{R}^{2}} f(x) d x-4 \pi t, \quad 0 \leq t<T .
$$

However, (1.5) shows that we must have equality in (3.28). Therefore $u$ is the desired solution. We can now use approximation arguments as in the proof of Theorem 1.2 to prove the result for any initial data $f \in L^{1}\left(\mathbf{R}^{N}\right)$.

To finish the proof of the Theorem, in the case that $\int_{\mathbf{R}^{2}} f(x) d x=\infty$, choose a sequence $f_{k} \uparrow f$, such that $\int_{\mathbf{R}^{2}} f_{k}<\infty$ and let $u_{k}$ be the maximal solution to the problem (1.1) with initial data $f_{k}$, as constructed above. The solution $u_{k}$ exists up to time $T_{k}=1 / 4 \pi \int_{\mathbf{R}^{2}} f_{k}$. Since, the sequence $u_{k}$ is increasing, the limit $u=\lim _{k \rightarrow \infty} u_{k}$ exists, and it is not hard to check that it is indeed a solution of (1.1) with initial data $f$, which exists up to time $T=\infty$. The proof of the Theorem is now complete.

\section{Proof of Theorem 1.3.}

We begin with a Lemma which constitutes the basic comparison argument which is needed for the proof of Theorem 1.3. Its proof is a straightforward consequence of our construction in Theorem 1.2. 
Lemma 4.1. Let $f_{1}, f_{2} \in L^{1}\left(\mathbf{R}^{2}\right), 0 \leq f_{1} \leq f_{2}$ and $0 \leq \mu_{2} \leq \mu_{1}<\infty$. Then, there exists solutions $u_{i}, i=1,2$ of the initial value problem (1.1) defined up to time $T_{i}=1 / 2 \pi\left(2+\mu_{i}\right) \int_{\mathbf{R}^{2}} f_{i}(x) d x$, satisfying

$$
\int_{\mathbf{R}^{2}} u_{i}(x, t) d x=\int_{\mathbf{R}^{2}} f(x) d x-2 \pi\left(2+\mu_{i}\right) t
$$

and such that

$$
u_{1} \leq u_{2}, \quad \text { on } \quad \mathbf{R}^{2} \times\left[0, T_{1}\right]
$$

Sketch of Proof. The construction in the proof of Theorem 1.2 shows that the solutions $u_{i}, i=1,2$ can be obtained as limits

$$
u_{i}=\lim _{j \rightarrow \infty} u_{i}^{R_{j}}
$$

where each $u_{i}^{R_{j}}$ solves the boundary value problem (3.1) on the cylinder $B_{R_{j}} \times[0, \infty)$ with initial data $u_{i}^{R_{j}}(x, 0)=f_{i}(x)$ and is obtained as a limit of regular solutions. Since $f_{1} \leq f_{2}$ and $R_{j}^{-\left(2+\mu_{1}\right)} \leq R_{j}^{-\left(2+\mu_{2}\right)}$, it follows by the maximum principle that

$$
u_{1}^{R_{j}} \leq u_{2}^{R_{j}}
$$

and therefore (4.1) holds.

Proof of Theorem 1.3. We will first show if $\phi$ is defined by (1.6), then it satisfies (1.7). The idea is similar to the one we just used for the proof of (1.4). Indeed, assume that at some point $\bar{t} \in[0, T)$ and some $\bar{h}>0$ we have the opposite inequality

$$
\frac{\phi(\bar{t}+\bar{h})-\phi(\bar{t})}{\bar{h}}<2
$$

From Theorem 1.1, we know that we can find a solution $v$ to (1.1) defined on $[\bar{t}+\bar{h}, \bar{t}+\bar{h}+T]$, whose initial data is $u(\cdot, \bar{t}+\bar{h})$, and where $T=(1 / 4 \pi) \int_{\mathbf{R}^{2}} u(x, \bar{t}+\bar{h}) d x$.

Thus, the function $w$ defined on $[\bar{t}, \bar{t}+h+T]$ by matching together $u$ and $v$ is a solution to (1.1) in this interval with initial data $u(\cdot, \bar{t})$. Now from (1.6) we can compute the integral of $u(\cdot, \bar{t})$ as

$$
\int_{\mathbf{R}^{2}} u(x, \bar{t}) d x=\int_{\mathbf{R}^{2}} f(x) d x-2 \pi \phi(\bar{t})
$$


and therefore from (1.3) we must have

$$
\bar{h}+T \leq \frac{1}{4 \pi}\left\{\int_{\mathbf{R}^{2}} f(x) d x-2 \pi \phi(\bar{t})\right\} .
$$

On the other hand, using (4.2) we deduce that

$$
\bar{h}+T=\bar{h}+\frac{1}{4 \pi}\left\{\int_{\mathbf{R}^{2}} f d x-2 \pi \phi(\bar{t}+\bar{h})\right\}>\frac{1}{4 \pi}\left\{\int_{\mathbf{R}^{2}} f d x-2 \pi \phi(\bar{t})\right\},
$$

the opposite of (4.3), contradiction showing the validity of (1.6).

We will show now the existence part of the Theorem. To construct a solution satisfying (1.6) we will approximate the function $\phi$ by a decreasing sequence of piecewise linear functions $\phi_{k}$, solve the IVP for such $\phi_{k}^{\prime} s$ and then pass to the limit.

For $k$ a positive integer, consider the partition $P_{k}=\left\{0=t_{0}<t_{1}<\ldots<\right.$ $\left.t_{2^{k}}=T\right\}$ of the interval $[0, T]$ into dyadic intervals, i.e., take $t_{i}=i T / 2^{k}$. Define $\phi_{k}$ on $\left[t_{i-1}, t_{i}\right), i=1,2, \ldots, 2^{k}$ as

$$
\phi_{k}(t)=\min \left(\phi_{k}\left(t_{i-1}\right)+\alpha_{i} t, K\right),
$$

with $\phi_{k}(0)=0$ and

$$
\alpha_{i}=\sup _{t_{i-1} \leq t \leq t_{i}} \phi^{\prime}(t)
$$

Since $\phi^{\prime}(t) \geq 2 \pi$ on $[0, T]$, we have that $\alpha_{i} \geq 2 \pi$. Let $T_{k}$ be the smallest $t$ such that $\phi_{k}(t)=K$. It is easy to check that the sequence $T_{k}$ is increasing with $T_{k} \uparrow T$, as $k \rightarrow \infty$. Moreover, we have

$$
\phi_{k+1} \leq \phi_{k} \quad \forall k \text {. }
$$

We will construct a weak solution $u_{k}$ of the $\operatorname{IVP}(1.1)$ on $\mathbf{R}^{2} \times\left(0, T_{k}\right)$, satisfying

$$
\int_{\mathbf{R}^{2}} u_{k}(x, t) d x=\int_{\mathbf{R}^{2}} f(x) d x-2 \pi \phi_{k}(t)
$$

Assune that $t_{\lambda} \leq T_{k}<t_{\lambda+1}$. We will define $u_{k}$ on $\left[t_{i-1}, t_{i}\right], i=1,2, \ldots, \lambda$ and $\left[t_{\lambda}, T_{k}\right]$ inductively. To begin, set $u_{k}(x, 0)=f(x)$. Then, set

$$
u_{k}(\cdot, t)=w_{i}\left(\cdot, t-t_{i-1}\right), \quad \text { on } \quad \mathbf{R}^{2} \times\left(t_{i-1}, t_{i}\right)
$$

where $w_{i}$ is a solution of the initial value problem $\partial w_{i} / \partial t=\Delta \log w_{i}$, $w_{i}(x, 0)=u_{k}\left(x, t_{i-1}\right)$, satisfying

$$
\int_{\mathbf{R}^{2}} w_{i}(x, t) d x=\int_{\mathbf{R}^{2}} u_{k}\left(x, t_{i-1}\right) d x-2 \pi \alpha_{i} t
$$


On the last interval $\left[t_{\lambda}, T_{k}\right]$, we define $u_{k}$ up to $T_{k}$. It is easy to check that each $u_{k}$ is indeed a solution of (1.1) on $\mathbf{R}^{2} \times\left(0, T_{k}\right)$. Also, from (4.5) and Lemma 4.1, we can conclude that

$$
u_{k+1} \geq u_{k}, \quad \text { on } \mathbf{R}^{2} \times\left(0, T_{k}\right)
$$

and therefore the limit

$$
u(x, t)=\lim _{k \rightarrow \infty} u_{k}(x, t)
$$

exists for all $(x, t) \in \mathbf{R}^{2} \times(0, T)$. Moreover, it follows from (4.6) and Monotone Convergence that $u(\cdot, t) \in L^{1}\left(\mathbf{R}^{2}\right), 0<t<T$ and that $u$ satisfies (1.6). To show that $u$ solves the equation in the distributional sense is standard as well as immediate. Hence, $u$ is the desired solution.

Remark. The condition that $\phi$ be $C^{1}$ in this result can be relaxed. In fact, one proceeds similarly in the case of a $\phi$ piecewise $C^{1}$. The present proof applies, more generally, to the case in which $\phi^{\prime}$ is Riemmann-integrable.

\section{References.}

[1] Aronson, D.G., Bénilan P., Régularité des solutions de l'équation de milieux poreux dans $\mathbf{R}^{n}$, C.R. Acad. Sci. Paris, 288 (1979), 103-105.

[2] Bertozzi, A.L., Pugh M., The lubrication approximation for thin viscous films : the moving contact line with a "porous media" cut off of Van de Waals interactions, preprint.

[3] Berryman and J.G., Holland, C.J., Asymptotic behaviour of the nonlinear differential equation $n_{t}=\left(n^{-1} n_{x}\right)_{x}$, J. Math Phys., 23 (1982), 983-987.

[4] Bertsch, M., Dal Paso, R., Ughi, M., Work in preparation.

[5] Bluman, G. and Kumei, S., On the remarkable nonlinear diffusion equation $\partial / \partial x\left[a(u+b)^{-2}[\partial u / \partial x]-(\partial u / \partial x)=0\right.$, J. Math. Phys. 21 (1980), 1019-1023.

[6] Dahlberg, B.E.G., Kenig, C.E., Non-negative solutions to fast diffusions, Rev. Mat. Iberoamericana, 4 (1988), 11-29.

[7] Daskalopoulos, P., del Pino M.A., On fast diffusion nonlinear heat equations and a related singular elliptic problem, Indiana Univ. Math. J. 43 (1994), 703-728. 
[8] Davis, S.H., DiBenedetto, E., Diller, D.,J., Some a-priori estimates for a singular evolution equation arising in thin film dynamics, preprint.

[9] Esteban, J.R., Rodriguez, A., Vazquez, J.L., A nonlinear heat equation with singular diffusivity, Arch. Rational Mech. Analysis, 103 (1988), 985-1039.

[10] de Gennes, P.G., Wetting: statics and dynamics, Reviews of Modern Physics, 57 No 3 (1985), 827-863.

[11] Hamilton, R., The Ricci flow on surfaces, Contemp. Math., 71, Amer. Math. Soc., Providence, RI, 1988, 237-262.

[12] Hamilton, R., The Harnack estimate for the Ricci flow, J. Differential Geometry, 37 (1993), 225-243.

[13] Herrero, M. and Pierre, M., The Cauchy problem for $u_{t}=\Delta u^{m}$ when $0<$ $m<1$, Trans. Amer. Math. Soc., 291 (1985), 145-158.

[14] Ladyzenskaja, O.A., Solonnikov, V.A., and Ural'ceva, N.N., Linear and quasilinear equations of parabolic type, Translations of Mathematical Monographs, 23 (1968).

[15] Rosenau, P., On a Fast and Super-Fast Diffusion, preprint.

[16] Rodríguez, A., Vázquez, J.L., A Well Posed Problem in Singular Fickian Diffusion, Arch. rational Mech. Anal., 110 (1990), 141-163.

[17] Vázquez, J.L., Nonexistence of solutions for nonlinear heat equations of fastdiffusion type, J. Math. Pures Appl., 71 (1992), 503-526.

[18] Wu, L.-F., A new result for the porous medium equation derived from the Ricci flow, Bull. Amer. Math. Soc., 28 (1993), 90-94.

[19] Wu, L.-F., The Ricci Flow on Complete $\mathbf{R}^{2}$, Communications in Analysis and Geometry, 1 (1993), 439-472.

RECEIVED JULY 5TH, 1995.

UNIVERSity of CALIForNia IRVINE

DEPARTMENT OF MATHEMATICS

IRVINE, CA 92717

AND

Departamento Matematicas

Facultad de Ciencias

UNIVERsidad DE ChILE 